\title{
Opportunistic Routing in Intermittently Connected Mobile P2P Networks
}

\author{
Shengling Wang, Member, IEEE, Min Liu, Member, IEEE, Xiuzhen Cheng, Senior Member, IEEE, \\ Zhongcheng Li, Member, IEEE, Jianhui Huang, Biao Chen, Member, IEEE
}

\begin{abstract}
Mobile P2P networking is an enabling technology for mobile devices to self-organize in an unstructured style and communicate in a peer-to-peer fashion. Due to user mobility and/or the unrestricted switching on/off of the mobile devices, links are intermittently connected and end-to-end paths may not exist, causing routing a very challenging problem. Moreover, the limited wireless spectrum and device resources together with the rapidly growing number of portable devices and amount of transmitted data make routing even harder. To tackle these challenges, the routing algorithms must be scalable, distributed, and light-weighted. Nevertheless, existing approaches usually cannot simultaneously satisfy all these three requirements. In this paper, we propose two opportunistic routing algorithms for intermittently connected mobile P2P networks, which exploit the spatial locality, spatial regularity, and activity heterogeneity of human mobility to select relays. The first algorithm employs a depth-search approach to diffuse the data towards the destination. The second one adopts a depth-width-search approach in a sense that it diffuses the data not only towards the destination but also to other directions determined by the actively moving nodes (activists) to find better relays. We perform both theoretical analysis as well as a comparison based simulation study. Our results obtained from both the synthetic data and the real world traces reveal that the proposed algorithms outperform the stateof-the-art in terms of delivery latency and delivery ratio.
\end{abstract}

Index Terms-Intermittently connected mobile P2P networks; opportunistic routing; human mobility

\section{INTRODUCTION}

Nowadays, billions of mobile devices are connected mainly through the assistance of infrastructures, which may often be undesirable due to high cost, lack of flexibility, and low utilization of the local wireless resources. Moreover, because infrastructures usually have limited wireless coverage and are vulnerable to nature disaster or other failures, only using this

Manuscript received 15 Feb 2012; revised 23 Jul 2012, accepted 15 Sep 2012.

Shengling Wang, Min Liu, and Zhongcheng Liu are with the Institute of Computing Technology, Chinese Academy of Sciences, Beijing, China. Email: \{wangshengling,liumin,zcli\}@ict.ac.cn

Xiuzhen Cheng is with the Department of Computer Science, The George Washington University, Washington DC 20052, USA. E-mail: cheng@gwu.edu

Jianhui Huang is with IBM China, Beijing, China. Email: jianhuih@cn.ibm.com

Biao Chen is with the Department of Computer Information Science, University of Macau, Macau, China. Email: bchen@umac.mo

This work has been supported by the National Basic Research Program of China (No. 2011CB302702 and No. 2012CB315802) and the National Natural Science Foundation of China (No. 61132001, No.61272475, No.61003225, No. 61070187, No.61003266 and No. 61070188), and the National Science Foundation of the US (CNS-0831852).

This work was completed when Xiuzhen Cheng visited University of Macau on her sabbatical leave. way may lead to network islands. As a result, a new networking paradigm named mobile peer-to-peer $(\mathrm{P} 2 \mathrm{P})$ networking has drawn enormous attention in recent years.

In a mobile $\mathrm{P} 2 \mathrm{P}$ network, mobile devices can communicate in a peer-to-peer fashion and self-organize in an unstructured style without the need of any infrastructure, making the local wireless connectivity better exploited. But under such a networking paradigm, data delivery is nontrivial [1]-[4] as end-to-end paths may not exist due to user mobility and/or the unrestricted switching on/off of the mobile devices. Moreover, the limited wireless spectrum and device resources (storage, computation capability, battery power, etc.), together with the rapidly growing number of portable devices and amount of transmitted data, make routing even harder. Thus an effective and efficient routing algorithm in intermittently connected mobile P2P networks should satisfy the following three design requirements:

1) Scalable. With the number of nodes in the network increases, the complexity of the algorithm as well as the information each node carries, maintains, and exchanges with others should not rapidly increase.

2) Distributed. Each node should determine its next hop independently, which means that no centralized routing decision/computation should get involved.

3) Light-weighted. Each node should incur low computation and storage overheads, which implies the simplicity of the routing algorithm.

As indicated in Section II, none of the existing routing algorithms could simultaneously satisfy all these three requirements. In this paper, we propose two opportunistic routing algorithms for intermittently connected mobile P2P networks. The first one takes a depth-search approach to diffuse the data towards its destination direction. This algorithm exploits the spatial regularity and spatial locality of the mobility of the device carriers, i.e. human beings ${ }^{1}$, to find relays. Because depthsearch delivers the data towards a single direction, adopting this strategy may miss better relays from other directions.

To make up this deficiency, a depth-width-search approach is proposed, which exploits not only the spatial regularity and spatial locality of human mobility, but also its activity heterogeneity. Thus, the data can be diffused towards not only the destination direction but also other directions determined by the actively moving nodes (activists) in order to find better relays. Since our algorithms require each node to maintain

\footnotetext{
${ }^{1}$ In this paper, we focus on the mobile devices carried by human beings because the number of such devices is prominent in the market.
} 
only the mobility characteristics of itself, which is a relatively stable piece of information in a dynamically mobile environment, they scale well in network size and incur low burden on each node. Moreover, our algorithms are distributed in nature as they involve no centralized decision/computation.

Our design is inspired by the following observations: the mobility of the mobile devices is mainly controlled by their carriers, the human beings, and the mobility of human beings is driven by their sociality, which is stable in long term. Thus by exploiting the stable characteristics of human mobility in both the time domain and the space domain, the relay selection can be simplified and the effectiveness as well as the efficiency of routing decisions can be guaranteed.

The rest of the paper is organized as follows. The related work is presented in Section II. Section III introduces the human mobility characteristics exploited in our algorithms and presents our routing model. The corresponding algorithms are detailed in Section IV. Section V provides our theoretical analysis on the proposed algorithms and the corresponding numerical validation. The simulation study is reported in Section VI. This paper is concluded by Section VII.

\section{RELATED WORK}

Existing routing algorithms for intermittently connected mobile P2P networks can be classified into two categories: deterministic and stochastic. Deterministic approaches [5]-[9] provide deterministic routing decisions assuming that some kinds of network connectivity information are known a priori. For example, Jain et al. [5] modify the Dijkstra's algorithm to compute the routes when the network connectivity patterns are known. In [6], a graph indexing system is proposed to minimize the long range communication cost for multicast, given the trajectories of moving peers and traffic demand are known in advance. By assuming that the contact rate between any two nodes in the network is available, [7] formulates a unified Knapsack problem for relay selection to support multicast, [8] studies the co-evolution of content popularity and delivery, and [9] proposes a data dissemination algorithm to select a relay in light of its social contact patterns and interests.

However, it is challenging to obtain the network connectivity information due to the uncertainty and dynamism of intermittently connected mobile P2P networks. Hence deterministic approaches are hard to implement in practice. This stirs the research of stochastic approaches [10]-[16], which are summarized as follows.

Gao et al. [10] exploit the transit contact pattern for each node, through which a node with a higher contact chance is selected as a relay. BUBBLE rap [11] combines the knowledge of the community structure with node centrality to make forwarding decisions. If the current relay does not meet a node whose community is the same as that of the destination, it selects the next hop according to the global centrality of the encountered nodes; if meets, it then considers the local centrality. SimBet routing [12] computes each node's centrality within the network and its social similarity to the destination for relay selection. Dang et al. [13] propose a cluster-based routing protocol, where a cluster is formed and a gateway is selected based on the nodal contact probabilities. Nodes within the same cluster use direct transmissions to communicate. When two nodes belonging to different clusters want to communicate, their gateways must relay the data.

The schemes mentioned above [10]-[13] all infer some kind of network connection information through the history nodal contacts. Epidemic [14], on the other hand, selects relays randomly without inferring any network connection information. To enhance the delivery ratio, Epidemic disseminates a large number of copies of each data, which incurs a heavy communication overhead. To trade off between the communication overhead and the delivery ratio, the binary spray algorithm [15] and the utility-based spray algorithm [16] are proposed. The binary spray algorithm employs a copy quota $Q$ to limit the maximum number of copies spread in the network. If a node holding the data meets another one without the data and the copy quota of this node is larger than 1, it delivers the data to the other node with half of the copy quota and keeps the other half for itself. The utilitybased spray algorithm [16] requires each node $i$ to maintain a utility function $U_{i}(j)$ for every other node $j$ in the network and selects relays according to the utilities of the nodes.

We claim that none of the existing algorithms simultaneously satisfies the three design requirements described in Section I for intermittently connected mobile P2P networks. For example, [5] is a centralized scheme, whose route computation complexity increases rapidly with the increase of the network size; though [7], [9], [10] are distributed, the computation overhead on each relay is high and increases rapidly when the number of nodes in the network increases; [13] selects cluster heads as gateways, resulting in the potential communication bottleneck problem; [10], [12], [16], [17] force each node maintain an entry for every other node in the network, thus scaling badly in network size; [11] needs to detect the community before relay selection, while the complexity of community detection increases exponentially with the network size; [14] adopts the flooding policy to deliver the data, which makes the delivery cost dramatically increase with the increase of the network size; though [15] limits the copy quota for each data, a large copy quota is needed for a good delivery ratio due to its simple relay selection strategy, again resulting in a poor scalability.

On the other hand, our proposed algorithms scale well and incur low overhead because the information maintained at each node is relatively stable and the relay selection is nodebased rather than node-relationship-based, which implies that our algorithms require no frequent information updates and the overhead on each node does not increase rapidly with the increase of the number of the nodes in the network. In addition, our algorithms are distributed because they involve no centralized routing decision/computation and communication hub. These advantages are achieved from exploiting the characteristics of human mobility, which will be introduced in the following section. 


\section{Motivation And Routing Model}

In this section, we first summarize the characteristics of human mobility that motivates our algorithm design and then detail our routing model.

\section{A. Characteristics of Human Mobility}

Our algorithms exploit three characteristics of human mobility introduced in our prior work [18] to simplify the relay selection for effective and efficient routing decisions. These characteristics are briefly reviewed in this section for completeness. They are obtained by analyzing a real world dataset, i.e. the Dartmouth College's wireless local area network (WLAN) traces [19]. This trace data records when each node connects to or disconnects from which access point (AP) in Dartmouth College during 2001 to 2004.

We analyze the data from the following four months: $09 / 21 \sim 10 / 20$ and $10 / 21 \sim 11 / 19$ in 2003, and $01 / 28 \sim 02 / 26$ and $04 / 20 \sim 05 / 19$ in 2004, during which about 5346 6052 mobile nodes roam among 532 543 APs. These four months are selected because their records are integral and the behaviors of the users are regular (no special events and vacations). Our analysis reveals the following human mobility characteristics:

i) Human mobility demonstrates a high degree of spatial regularity. This indicates that each node has a significant probability of returning to a few highly frequently visited places. As shown in Figure 1(a), nodes are found at their first two preferred places with a probability over $70 \%$.

ii) Human mobility exhibits spatial locality. In other words, people usually move within a local region. Figure 1 (b) reports the probability density function of the average displacement of all mobile nodes from their corresponding centers (most frequently visited places). According to Figure 1(b), the probability of moving away from its center for a node decreases sharply with the increase of the displacement, exhibiting spatial locality of human mobility.

iii) The activities of human mobility are heterogeneous. In real world, different nodes have different mobility activities characterized by the number of different locations visited within a given time. From Figure 2(a), we observe that about $52 \% \sim 58 \%$ nodes visit less than 10 APs; about $20 \%$ nodes visit 10 20 APs; about $2 \% \sim 3 \%$ nodes visit 40 50 APs; some nodes visit more than $80 \mathrm{APs}$; and one or several nodes even visit more than 200 APs. Different mobility activities result in different chances of contacting with others. Figure 2(b) indicates that with the increase of the mobility activities, the average number of nodes one can contact increases rapidly.

It is worth noting that similar human mobility characteristics are also observed from other real world datasets. For example, the dataset obtained by tracking the trajectories of 100,000 anonymized mobile phone users for a six-month period [20] indicates that nodes stay in their first two preferred places with a probability over $40 \%$, demonstrating the spatial regularity of human mobility; A three-month record [21] capturing the mobility patterns of 50,000 nodes chosen from about 10 million anonymous mobile phone users shows that a user is found with the probability of $70 \%$ in his/her most visited location during the observed hour; in addition, most individual's daily activity

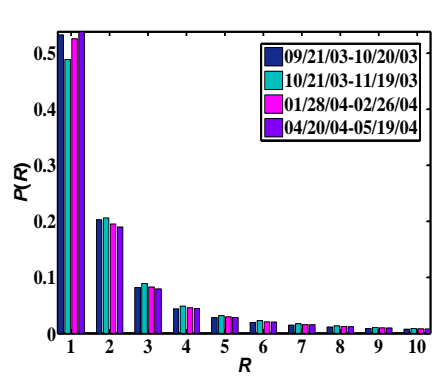

(a)

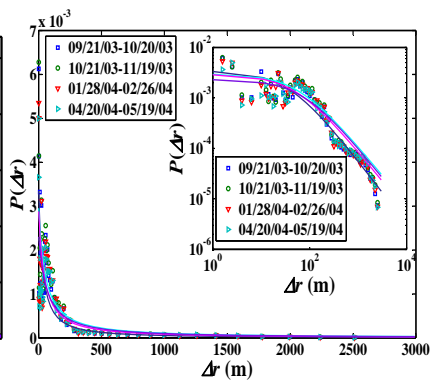

(b)
Figure 1: [18] (a) A Zipf plot showing the average probabilities of frequently visited places in four months. The $\mathrm{X}$ label $(R)$ is the rank of the APs listed in the order of visiting frequencies, and the Y label is the probability of $R$. (b) The probability density function of average displacement over all nodes. We use the first frequently visited AP as the center place of a node, and count the probability of each node's displacement $(\triangle r)$ from its center. The axes graph is the logarithmic plot for better illustration.

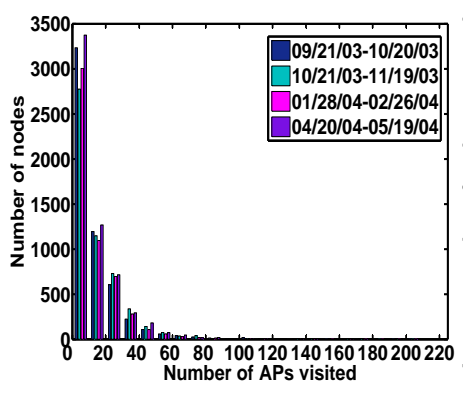

(a)

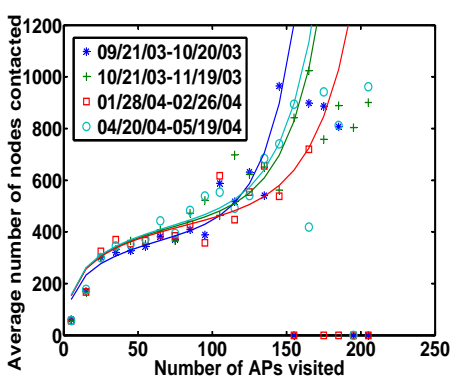

(b)
Figure 2: [18] (a) reports how many nodes visit how many different APs in 30 days and (b) shows how the average number of nodes a node contacts changes with the number of different APs visited by the node within 30 days.

is confined to a limited neighborhood of 1 to $10 \mathrm{~km}$ and a few users cover hundreds of kilometers. This trace data [21] verifies the spatial regularity and spatial locality of human mobility. The trace data [22] collecting approximately 275 freshmen PDA users for 11 weeks reveals that $50 \%$ users visit 21 APs or more, $20 \%$ users visit 56 APs or more, $10 \%$ users visit 71 APs or more, and in extreme cases, some users visit over 130 APs, which demonstrates the heterogeneous activity of human mobility.

By taking advantage of the human mobility characteristics summarized in this section, we design two routing algorithms to disseminate the data through depth or/and width searches as described in Section IV.

\section{B. Routing Model}

In this section, we introduce our network model in detail. The whole intermittently connected mobile P2P network $(\Omega)$ is divided into multiple zones $\left(Z_{i}\right)$, with $\cup Z_{i}=\Omega$ and $\cap Z_{i}=\emptyset$. 
These zones can have any shape. Each zone $Z_{i}$ is identified by its center coordinates $\left(x_{i}, y_{i}\right)$.

There are many methods for a node to figure out the center coordinators of a zone. For example, the center coordinates of a zone can be broadcasted by the access points or access routers in an infrastructure-based network; or they can be determined based on a mapping function if the node is aware of its own physical location.

Definition 3.1 (Home): The home of node $i$, denoted by $H_{i}$, is the set of zone(s) it visits at high frienquencies.

There exist two simple strategies for a node to determine its home: i) a node can statically configure the zones it usually visits as its home; ii) it can dynamically add a zone to its home once the visiting frequency of the zone is larger than a given threshold. Similarly, a zone can be deleted from a node's home either statically or dynamically.

Definition 3.2 (Neighbor set): The neighbor set of node $i$, denoted by $N_{i}$, is the set of nodes that can communicate directly with $i$.

We assume that any two nodes located at the same zone can communicate directly with each other. Therefore, all the nodes covered by the zone where $i$ resides belong to $N_{i}$. More generally, $N_{i}$ includes the nodes in a neighboring zone that can communicate with $i$ directly. Note that $i \notin N_{i}$.

Definition 3.3 (Activist): If the number of different zones visited by a node within a unit time exceeds a threshold, the node is called an activist.

Definition 3.4 (Distance between homes): The distance between the homes of two node $i$ and $j$ is the minimum distance between any two zones of $H_{i}$ and $H_{j}$, i.e., $\left\|H_{i}-H_{j}\right\|=$ $\min \left\{\left\|Z_{i}-Z_{j}\right\| \mid Z_{i} \in H_{i}, Z_{j} \in H_{j}\right\}$.

Definition 3.5 (Home node): Node $i$ is called a home node of a packet if the distance between the home of $i$ and that of the destination of the packet is 0 , i.e., $\left\|H_{i}-H_{d}\right\|=0$.

In our model, once two nodes contact, they need to exchange their home information. If a node is an activist, it should indicate this status.

\section{Our Algorithms}

In this section, we propose two algorithms to select relays for routing in intermittently connected mobile P2P networks exploiting the three characteristics of human mobility introduced in Section III-A.

\section{A. Depth-Search}

As shown in Section III-A, human mobility exhibits a high degree of spatial regularity and spatial locality. Thus, the destination appears in the zones or near the zones of its home with a high probability. Therefore to achieve a high delivery ratio, it is better to select as relays the nodes that usually visit such zones.

According to the above idea, we propose the depth-search algorithm, which selects as relays the nodes whose homes' distances to that of the destination are within a given threshold $L$ (Line 9). In particular, when $L=0$, the depth-search algorithm only selects home nodes as relays. The pseudo-code of this algorithm is given in Algorithm 1, where $Q_{j}$ is the copy

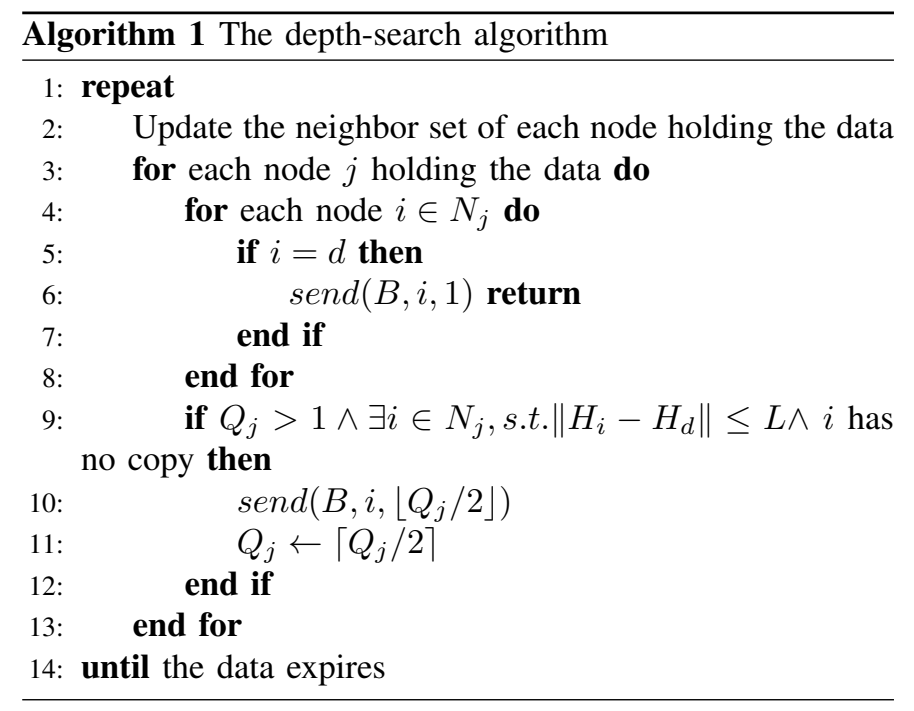

quota of node $j$ and function $\operatorname{send}(B, i, Q)$ sends data $B$ to node $i$ with copy quota $Q$. The copy quota of a node limits the number of copies delivered to other relays. If $Q=1$, no copy is allowed to deliver to other relays.

Algorithm 1 selects a relay according to the following policy: for a node $j$ currently holding the data, if it meets the destination $d$, it should deliver the data to $d$ directly (Lines $3-8$ ); if it meets a node satisfying the relay condition and its copy quota is larger than 1 , it delivers the data to the node and gives the node $\left\lfloor Q_{j} / 2\right\rfloor$ copy quota, leaving with itself $\left\lceil Q_{j} / 2\right\rceil$ copy quota (Lines 9-12).

\section{B. Depth-Width-Search}

The depth-search strategy exhibits a single-direction search, which may miss better relays from other directions. To make up this deficiency, we propose the depth-width-search approach, which not only exploits the spatial regularity and the spatial locality of human mobility, but also the activity heterogeneity of human mobility. The underlying rationale lies in that the nodes with high mobility activities usually have high chances to meet with others, and thus the probability that it meets better relays is high.

Therefore the depth-width-search approach selects as relays the activists besides the nodes whose homes are near to the home of the destination (Line 9). The pseudo-code of this algorithm is presented in Algorithm 2.

It is worth noting that in a large network with sparse mobile nodes, the source may spend a long time to find a node satisfying the relay condition. In such a scenario, our algorithms suffer from a so-called slow start phase, which largely affects the data diffusion speed in the network. To overcome this problem, we can slightly modify the algorithms by adding the following consideration: if the source does not meet a node satisfying the relay condition for a given amount of time, it selects as a relay the node from its neighbor set whose home is the nearest to the home of the destination. There also exist other variations that can further enhance the performance of our algorithms, which will not be detailed in this paper due to space limitations. 

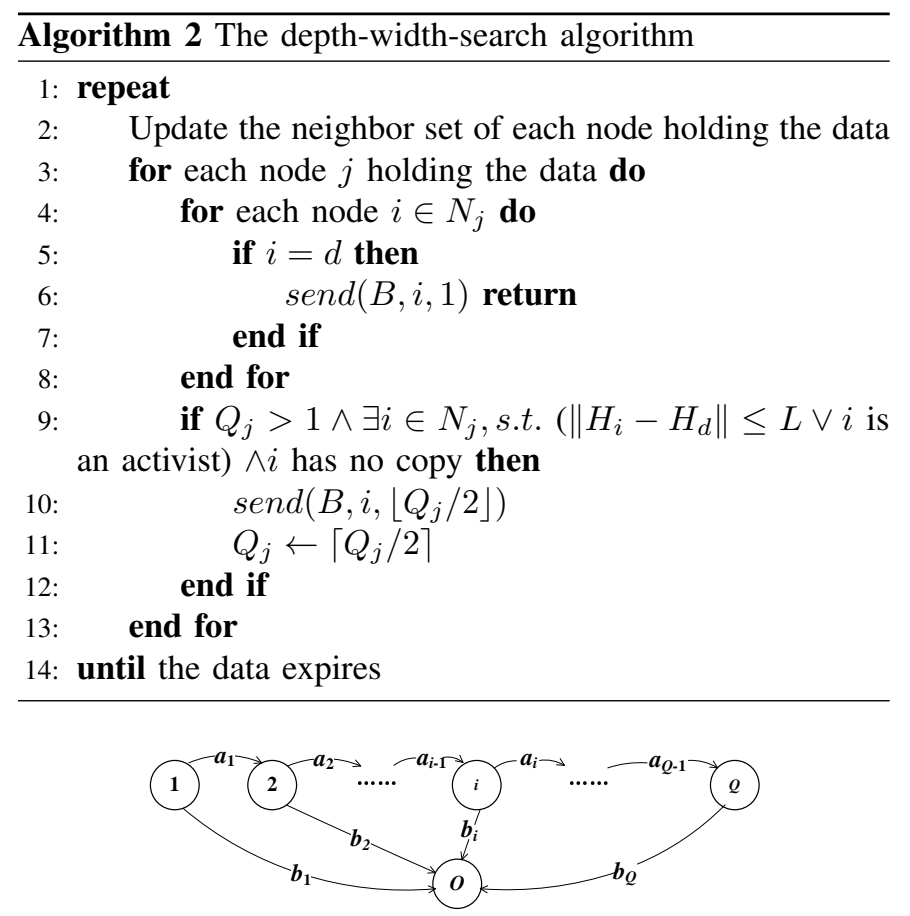

Figure 3: A continuous time Markov model for analyzing the data delivery.

\section{THEORETICAL ANALYSiS AND NUMERICAL VALIDATION}

In this section, we elaborate on the theoretical analysis of our algorithms and provide simulation results for validation purpose.

\section{A. Theoretical Analysis}

We assume that there are $Z$ zones and $U$ nodes in the network. The maximum number of copies a data packet can have in the network is denoted by $Q$. Note that in our analysis we omit the wireless transmission delay when calculating the data delivery latency because it is negligible compared to the duration that two nodes contact ${ }^{2}$.

Similar to [24], we employ a continuous time Markov model to analyze our algorithms. As shown in Figure 3, a state $i$ indicates that there are $i$ relays in the network and the absorbing state $O$ represents the state at which the data is delivered to the destination. We let the transfer rate from state $i$ to state $i+1$ be $a_{i}$ while to state $O$ be $b_{i}$. In the following, we elaborate on how to derive $a_{i}$ and $b_{i}$.

Definition 5.1 (Potential relay of depth-search): A node $u$ with no copy of the data is a potential relay of the depthsearch algorithm if $\left\|H_{u}-H_{d}\right\| \leq L$.

Definition 5.2 (Potential relay of depth-width-search):

A node $u$ with no copy of the data is a potential relay of the depth-width-search algorithm if it satisfies one of the following two conditions: i) $\left\|H_{u}-H_{d}\right\| \leq L$, and ii) it is an activist.

Definition 5.3 (Distance between a zone and a home): The distance between a zone $i$, i.e. $Z_{i}$, and the home of a

\footnotetext{
${ }^{2} \mathrm{~A}$ similar assumption is adopted by [23].
}

node $j$, i.e. $H_{j}$, is the minimum distance between $Z_{i}$ and any zone in $H_{j}$, i.e., $\left\|H_{j}-Z_{i}\right\|=\min \left\{\left\|Z_{j}-Z_{i}\right\| \mid Z_{j} \in H_{j}\right\}$.

Lemma 5.1: The probability $\left(\Pi_{d}\right)$ that a node becomes a potential relay of the depth-search algorithm when there are $i$ copies of a data packet in the network is $\Pi_{d}=\frac{(U-i-1) O_{L}}{(U-1) Z}$, where $O_{L}$ is the number of zones whose distances to the home of the destination are within $L$.

Proof: When there are $i$ copies in the network, the probability that any node except the destination has no such copy is $\frac{U-i-1}{U-1}$. For an arbitrary node, the probability that the distance from its home to that of the destination is within $L$ is $O_{L} / Z$. According to Definition $6.1, \Pi_{d}=\frac{(U-i-1) O_{L}}{(U-1) Z}$.

According to Definition 4.2, if the number of different zones visited by a node within a unit time is beyond a threshold, the node is an activist. Given this threshold, the number of activists in the network can be determined. We assume that there are $m$ activists in the network. Then with a proof similar to that of Lemma 5.1, we have

Lemma 5.2: The probability $\left(\Pi_{d w}\right)$ that a node becomes a potential relay of the depth-width-search algorithm when there are $i$ copies of the data in the network is $\Pi_{d w}=\frac{U-i-1}{U-1} \cdot(1-$ $\left.\left(1-\frac{O_{L}}{Z}\right)\left(1-\frac{m}{U}\right)\right)$.

Let $\Pi$ be the probability that a node is a potential relay of our algorithms. Then in depth-search, $\Pi=\Pi_{d}$ while in depth-width-search, $\Pi=\Pi_{d w}$. Let the average rate at which any node contacts with others be $\lambda$.

Lemma 5.3: When there are $i$ nodes except the destination holding copies of a data packet, the data delivery rate $\left(b_{i}\right)$ can be computed by $i \lambda /(U-1)$.

Proof: When $i$ nodes have copies of the data in the network, the probability that a node except the destination has a copy is $i /(U-1)$. Since the rate of the destination contacting with other nodes is $\lambda$, we have $b_{i}=i \lambda /(U-1)$.

To compute $a_{i}$, we have to address the following question: when there are $i$ relays in the network, how many of them can forward their copies to other nodes? To answer this question, we need first to investigate another question: when there are $j$ relays in the network and the maximum copy-limitation is $Q$, how do these $j$ relays share the copy quota $Q$ ? According to [24], this question is very similar to the integer partition problem [25], which asks for the number of possible partitions of a given integer. Let $N(Q \mid j)$ be the average number of nodes among the $j$ nodes that can forward their copies to other nodes when the maximum copy-limitation is $Q$. According to [25], $N(Q \mid j)$ can be calculated by:

$$
N(Q \mid j)= \begin{cases}0 & j=0 \\ \frac{\sum_{s=1}^{j} s M(Q-j \mid s)}{M(Q \mid j)} & \text { otherwise }\end{cases}
$$

with

$$
M(Q \mid j)= \begin{cases}0 & Q<j \\ 1 & Q=j \\ \sum_{y=1}^{j} M(Q-j \mid y) & Q>j\end{cases}
$$


is:

$$
a_{j}= \begin{cases}1 & j=0 \\ N(Q \mid j) \Pi \lambda & 1 \leq j<Q \\ 0 & j=Q\end{cases}
$$

Proof: Obviously, $a_{0}=1$ and $a_{Q}=0$. When there are $1 \leq j<Q$ relays in the network, in average $N(Q \mid j)$ relays have the capacity (whose copy quota is larger than 1 ) to send the data to other nodes. Since any relay meets other nodes with the rate of $\lambda$ and each node it meets has the probability of $\Pi$ to satisfy the relay condition, $a_{j}=N(Q \mid j) \Pi \lambda$ when $1 \leq j<Q$.

Theorem 5.1: The probability density function of the data delivery latency, denoted by $f_{O}(t)$, is $\sum_{i=1}^{Q} b_{i} \otimes_{j=1}^{i}$ $a_{j-1} e^{-\left(a_{j}+b_{j}\right) t}$.

Proof: Let $F_{i}(t)$ and $F_{O}(t)$ be the distributions of the probabilities at which the state is in $i$ and $O$, respectively. According to the Kolmogorov's equation [26], we have:

$$
F_{i}^{\prime}(t)= \begin{cases}-\left(a_{i}+b_{i}\right) F_{i}(t) & i=1 \\ -\left(a_{i}+b_{i}\right) F_{i}(t)+a_{i-1} F_{i-1}(t) & i=2, \ldots, Q-1 \\ -b_{i} F_{i}(t)+a_{i-1} F_{i-1}(t) & i=Q \\ \sum_{j=1}^{Q} b_{j} F_{j}(t) & i=O\end{cases}
$$

Since $F_{1}(0)=1$ and $F_{i}(0)=F_{O}(0)=0$, through Laplace and inverse Laplace transforms, the probability density function of the data delivery latency, $f_{O}(t)$, can be computed by $(5)$, where $\otimes$ represents the convolution operation.

$$
f_{O}(t)=\sum_{i=1}^{Q} b_{i} \otimes_{j=1}^{i} a_{j-1} e^{-\left(a_{j}+b_{j}\right) t}
$$

\section{B. Numerical Validation}

The probability density function $f_{O}(t)$ of the data delivery latency is very important because both the average delivery latency and the average delivery ratio can be deduced from it. In fact, the average delivery latency can be calculated by $\int_{0}^{\infty} t f_{O}(t) d t$ while the average delivery ratio within an observation duration $T$ is $\int_{0}^{T} f_{O}(t) d t$. In this subsection, we provide simulation results to validate the probability density function $f_{O}(t)$ of the data delivery latency.

Our simulated network contains $50 \times 50$ zones and 100 mobile nodes. For these 100 mobile nodes, we randomly allocate serval zones to each of them as their homes. They move from one zone to another zone of their homes randomly. The most ten active mobile nodes are selected as the activists. We set $Q=10$ or 20 and $L=20$, which will be explained in the following section. To validate $f_{O}(t)$, we employ 10000 different random seeds to produce 10000 different simulation instances. The reported $f_{O}(t)$ is the statistical result of the 10000 simulation instances.

In this numerical analysis, the rate $\left(\lambda_{i}\right)$ at which each node $i$ contacts with others is set according to the statistical value obtained from the simulation study, through which the average rate at which each node contacts with others $\left(\lambda=\frac{\sum_{i=1}^{U} \lambda_{i}}{U}\right)$ can be obtained. Figure 4 reports the results of the simulated
$f_{O}(t)$ and the numerical value obtained from the theoretical analysis, from which we observe that the trends of both results are matched.

In the next section, we further evaluate our algorithms using both the synthetic data described above and the real world trace data. We do not use the real world trace data for validating $f_{O}(t)$ because the rate at which each node contacts with others is quite different (see Figure 2(b)). Thus the $\lambda$ computed by normalizing $\lambda_{i}$ deviates significantly from the actual rate at which each node contacts with others. Though normalizing $\lambda$ results in a large deviation, it is unavoidable when modeling the data diffusion speed. We would like to emphasize that this is a commonly-accepted method to perform similar analysis [24], [27], [28].

\section{Simulation RESUlts}

In this section, we evaluate the performance of our algorithms based on the real world trace data and the synthetic data introduced in Section V-B. We will compare our algorithms with binary spray [15] (labeled by Binary) and BUBBLE rap [11] (labeled by BUBBLE).

In the binary spray algorithm, a node with a copy quota larger than 1 for a packet delivers its data to another node it meets with half of its copy quota and leaves itself with the other half if the encountered node has no copy of the data.

The BUBBLE rap algorithm is a famous opportunistic routing algorithm for intermittently connected mobile $\mathrm{P} 2 \mathrm{P}$ networks. As introduced in Section II, BUBBLE combines the knowledge of the community structure with node centrality to make forwarding decisions. Hence, BUBBLE needs community detection before selecting relays. Similar to [11], we employ the $K$-CLIQUE [29] method to detect community when implementing BUBBLE in our study.

To utilize the $K$-CLIQUE method, we must determine the edge between any two nodes in advance. Again we employ the method adopted by [11]: there is an edge between any two nodes if their contact duration is greater than a threshold. To determine the threshold, we use the traditional method for analyzing complex networks ${ }^{3}$ : the threshold is a value slightly greater than the clique percolation threshold. In other words, our threshold is selected to partition the whole network into multiple communities rather than a big community plus some fragments. In addition, we set $K=4$, which is an empirical value typically adopted by community detection.

Note that BUBBLE is a single-copy multiple-relay algorithm while ours are multiple-copy multiple-relay ones. Therefore for a fair comparison, we extend BUBBLE to support multiple-copy delivery: when a source sends a data packet to a destination, it sends $Q$ copies, with each being delivered according to the strategy in the original BUBBLE rap algorithm. Once a copy arrives in the destination, the delivery of the data terminates. It is worth noting that if a node has a copy of the data, it will be ignored in the multiplerelay multiple-copy version even though it is deemed optimal according to the original BUBBLE strategy. In another word,

\footnotetext{
${ }^{3}$ The intermittently connected mobile P2P network is a typical complex
} network. 


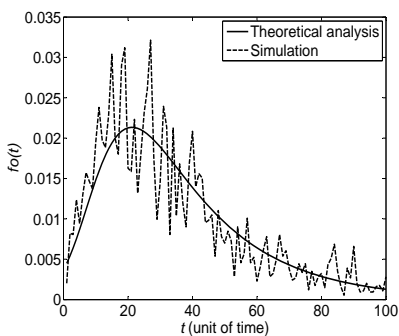

(a)

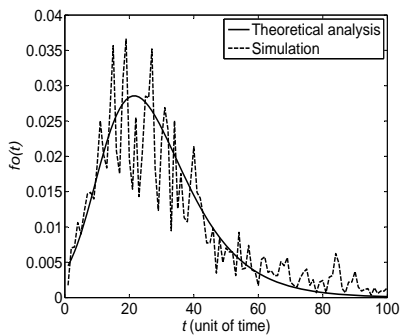

(b)

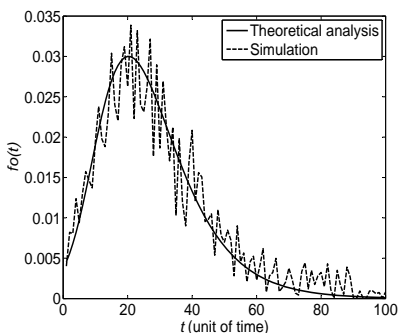

(c)

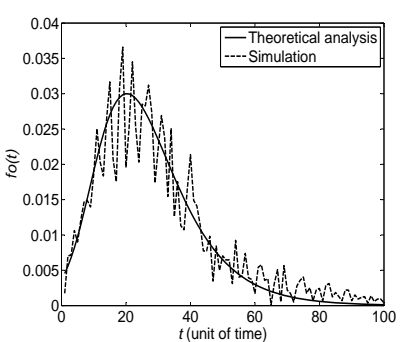

(d)

Figure 4: Theoretical results vs. simulation results: (a) Depth-search with $Q=10$; (b) Depth-search with $Q=20$; (c) Depthwidth-search with $Q=10$; (d) Depth-width-search with $Q=20$.

a relay cannot receive duplicate copies in the multiple-relay multiple-copy version.

The performance metrics for our simulation study include the delivery ratio and the delivery latency. It is less meaningful to compare the delivery latency of two algorithms when their successful communication pairs are not the same. The reason is that the delivery latency of the communication pairs that succeed in one algorithm but fail in another may worsen the average delivery latency of the algorithm with a higher delivery ratio $^{4}$. Hence in the following, we only compare the average delivery latency of the communication pairs that succeed in all algorithms under our comparison study. Finally, for simplicity, we assume that the lifetime of a data packet equals the simulation duration.

\section{A. Simulation Results Over the Synthetic Data}

In this subsection, we evaluate our algorithms based on the synthetic data introduced in Subsection V-B. We select 100 source-destination pairs from the 100 mobile nodes and test the cases when $L=0,10,20$, and 30 . Our results indicates that $L=20$ yields the best performance because this setting provides the best tradeoff between the number of relays and the quality of relays.

Figures 5 and 6 respectively report the delivery ratio and average latency of the four algorithms. We observe that our algorithms outperform Binary in all settings and BUBBLE in most cases. The performance of Binary is the worst because it selects relays only based on whether or not the encountered node has a copy of the data and ignores other key properties of nodes. The performance gap between BUBBLE and our algorithms is resulted from the fact that the former selects as relays the nodes that usually contact the destination through communities while ours select relays based on the zones they usually visit. The contacts among the nodes in a community are usually indirect, which may lead to unsatisfactory performance. For example, if nodes $a, b$, and $c$ belong to the same community but $a$ usually contacts with $b$, who usually contacts with $c$. In the case that the destination is $c$, and the centrality of $a$ is higher than that of $b$, a data packet will be delivered to $a$ rather than $b$. Then $c$ may not receive the data because it cannot contact $a$ directly.

\footnotetext{
${ }^{4}$ The source-destination pair that is hard to communicate successfully usually has a longer delivery latency.
}

From Figures 5 and 6 we also observe that the delivery ratio of the depth-search is slightly higher than that of the depth-width-search, while the average latency of the latter is smaller than that of the former. This is because the depthwidth-search also employs activists as relays, which have a smaller probability of meeting the destination than the home nodes or the nodes whose homes are near to that of the destination, leading to a slight loss in terms of the delivery ratio. However, activists may meet better relays from other directions, resulting in a lower delivery latency.

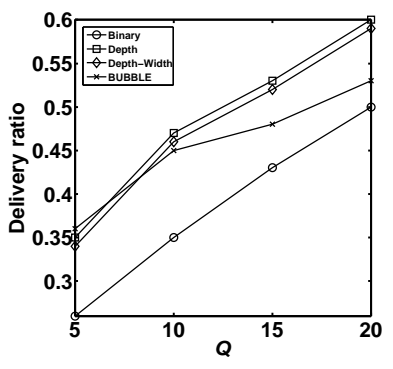

Figure 5: Delivery ratio comparison over the synthetic data.

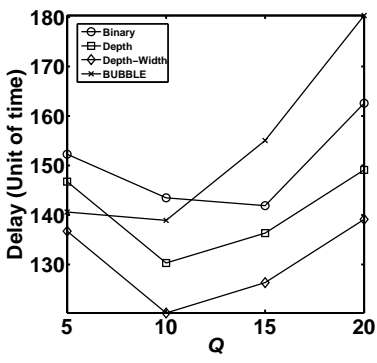

Figure 6: Average delay comparison over the synthetic data.

\section{B. Performance Evaluation Over the Real-World Trace Data}

In this subsection, we evaluate our algorithms using the Dartmouth College mobility trace data [19]. We choose the data collected from $09 / 21 / 2003$ to $10 / 20 / 2003$ because in this period the records are integral and the nodes' behaviors are regular.

In our simulation study, each AP represents a zone, and any two nodes within the same zone can communicate directly. Because the trace data contains over 5000 mobile nodes and the running time increases rapidly with the increase of the number of mobile nodes, we limit the number of nodes to a manageable size. The same strategy is taken by [16], [30], [31], which also use the Dartmouth College trace data. Different from these existing literatures, we do not deliberately select the well-connected mobile nodes such as "good neighbors" [30] or "active nodes" [31] to construct the simulation topology. Instead, we select mobile nodes completely randomly from the 
Dartmouth College trace data to retain as much as possible the characteristics of the raw data.

Since the number of nodes in the network needs to be limited to a manageable size, we construct the simulation scenarios according to the following steps:. We first randomly choose 300 mobile nodes to construct a 300-node scenario, and select 100 source-destination pairs from these 300 mobile nodes. These 100 communication pairs remain unchanged during our simulation study. Then we add 100 newly selected random mobile nodes to the 300 -node scenario to get a 400 node scenario. Repeat this procedure we can construct a 500node scenario. For simplicity, we denote by $U=x$ the $x$-node scenario, where $U$ is the network size.

We have tested the performance of our algorithms when an activist is one of the 20,30,40 nodes that visit the largest number of different zones during the 30 -day period. We found that if the data is delivered to a node that is more active than others, the delivery ratio is improved. However, if the threshold for activist selection is increased, the number of relays is reduced, which decreases the delivery ratio. Our results indicate that selecting the top 30 active nodes results in the best performance. We also evaluate the performance of our algorithms on different home size, which is defined to be the number of zones in a home. Through extensive tests we found that when the largest home size is limited to $10 \%$ of the zones in the network, namely the average home size is about 10 , a trade-off between quality and quantity of the relays can be obtained.

Because the real world network is more complex than the synthetic one, finding a good $L$ for the Dartmouth trace data is quite challenging. Hence we investigate the performance of our algorithms for $L=0$ and $L=300$. Note that we choose these two $L$ values that differ significantly because the performances of our algorithms exhibit a small difference when the change of $L$ is not large as the real world network is large. Figure 7 illustrates the delivery ratios of our algorithms for $L=0$ and $L=300$, the BUBBLE rap algorithm, and the binary spray algorithm when $U$ varies. Because the number of communication pairs succeeding in all the six cases (two for each of our algorithms, two for BUBBLE, and two for Binary) is not large, we consider $L=0$ and $L=300$ separately when investigating the average delivery latency. Figures 8 and 9 report the average delivery latencies of the communication pairs succeeding in all the four algorithms for the cases $L=0$ and $L=300$, respectively.

According to Figure 7, the delivery ratios of our algorithms are higher than that of the binary spray algorithm in all cases. When $U=300$, the delivery ratios of our algorithms are also higher than that of the BUBBLE rap algorithm, and the gap reduces with the increase of $U$. However, as shown in Figures 8 and 9, the average delivery latencies of our algorithms are smaller than those of the BUBBLE rap algorithm and the binary spray algorithm in all cases. In addition, though the delivery ratio of the depth-search is slightly higher than that of the depth-width-search, the delay of the latter is smaller than that of the former.

It is worth noting that the storage costs of our algorithms are higher than that of the binary spray algorithm because we require each node to maintain the information of its home and whether it is an activist. However, these costs are valuable because our algorithms significantly outperform the binary spray algorithm.

Also note that the time complexity of our algorithms are lower than that of the BUBBLE rap algorithm because the latter needs to detect the community before relay selection. When using the $K$-CLIQUE method as in [11], the community detection mainly involves two steps: i) finding all the $K$ cliques; and 2) finding the communities by searching the adjacent $K$-cliques, where two $K$-cliques are adjacent if they share $K-1$ nodes. The first step determines the time complexity of community detection. According to [29], the complexity of identifying all $K$-cliques increases exponentially with the increase of the network size. However, the complexities of our algorithms increase only linearly with the increase of the network size. Moreover, $K$-CLIQUE is centralized, which does not fit the decentralization requirement of mobile $\mathrm{P} 2 \mathrm{P}$ networks.

\section{CONCLUSION}

In this paper, we propose two opportunistic routing algorithms for intermittently connected mobile P2P networks, with both taking advantage of the human mobility characteristics, namely the spatial regularity, the spatial locality, and the activity heterogeneity of human mobility, for enhancing the routing efficiency. The first one is a depth-search algorithm that diffuses the data toward the destination direction while the second one adopts the depth-width-search, which delivers the data toward both the destination direction and other directions guided by the activists. To validate the performance of our algorithms, we first perform a theoretical analysis, and then conduct a comparison based simulation study over both the synthetic data and the real world traces. The results indicate that our algorithms outperform the most related ones in terms of the delivery ratio and average delivery latency.

\section{REFERENCES}

[1] O. Yagan, D. Qian, J. Zhang, and D. Cochran, "Conjoining speeds up information diffusion in overlaying social-physical networks," Submitted to IEEE JSAC Special Issue on Network Science, 2013.

[2] W. Cheng, D. Wu, X. Cheng, and D. Chen, "Routing for information leakage reduction in multi-channel multi-hop ad-hoc social networks," in WASA, August 2012, pp. 31-42.

[3] S. Wang, M. Liu, X. Cheng, Z. Li, J. Huang, and B. Chen, "Hero a home based routing in pocket switched networks," in WASA, August 2012, pp. 20-30.

[4] Y. Duan, A. G. Bourgeois, and Z. Cai, "Delay efficient opportunistic routing in asynchronous multi-channel cognitive radio networks," in Submitted to IEEE INFOCOM, 2013.

[5] S. Jain, K. Fall, and R. Patra, "Routing in a delay tolerant network," in SIGCOMM '04: the conference on Applications, technologies, architectures, and protocols for computer communications, New York, USA, 2004, pp. $145-158$.

[6] M. Mongiovì, A. Singh, X. Yan, B. Zong, and K. Psounis, "Efficient multicasting for delay tolerant networks using graph indexing," in INFOCOM, Orlando, Florida USA, 2012, pp. 1386 - 1394

[7] W. Gao, Q. Li, B. Zhao, and G. Cao, "Multicasting in delay tolerant networks: a social network perspective," in MobiHoc, New Orleans, USA, 2009, pp. 299-308.

[8] S. Venkatramanan and A. Kumar, "Co-evolution of content popularity and delivery in mobile p2p networks," in INFOCOM, Orlando, Florida USA, 2012, pp. 3006-3010. 


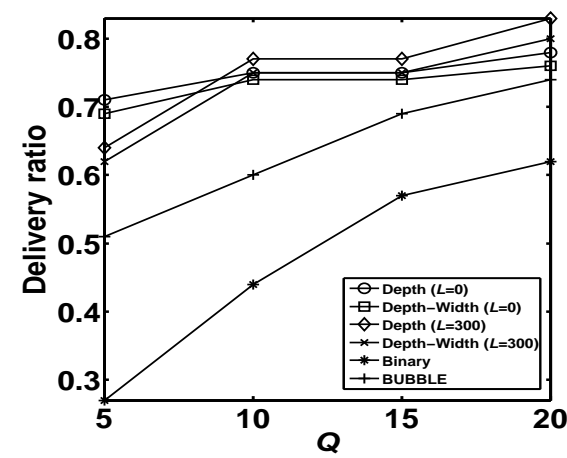

(a) $U=300$

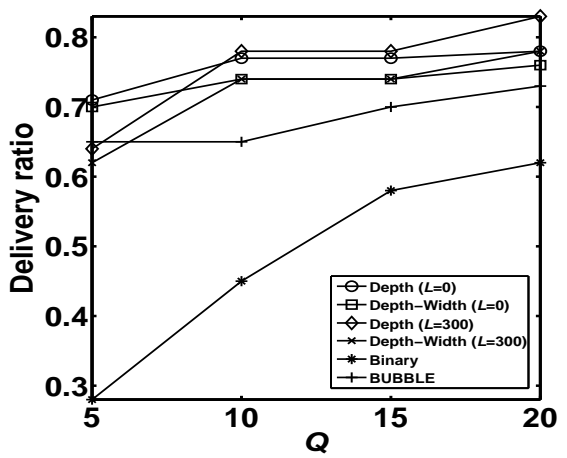

(b) $U=400$

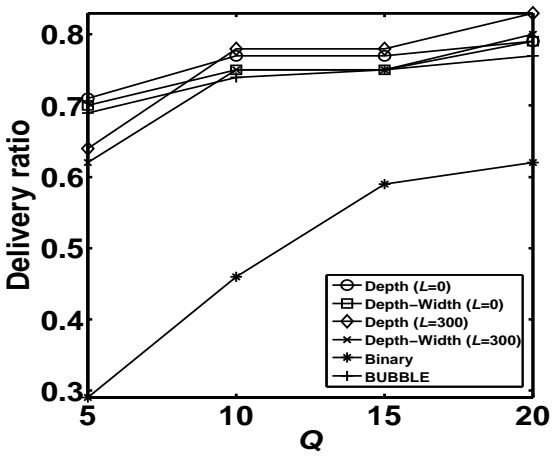

(c) $U=500$

Figure 7: Delivery ratio comparison using the real world trace data.

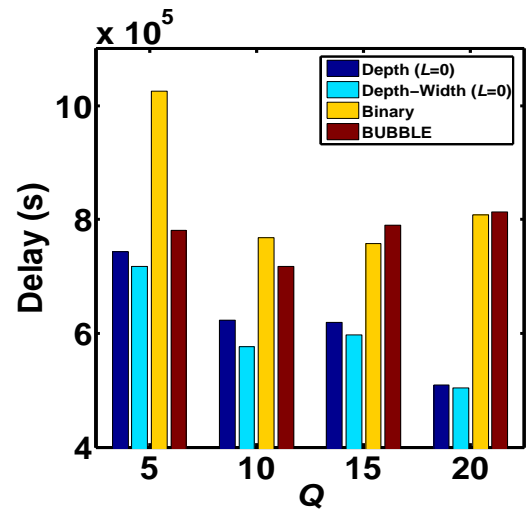

(a) $U=300$

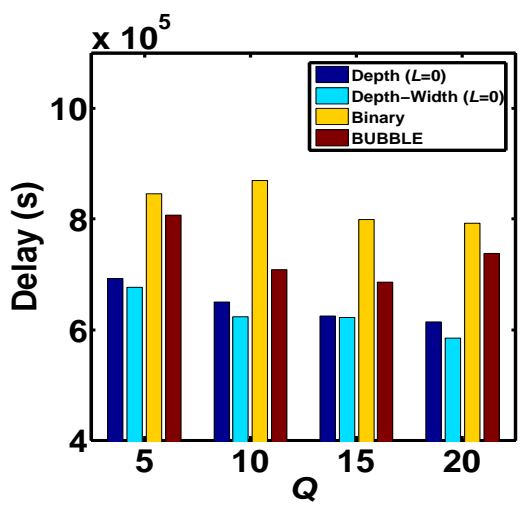

(b) $U=400$

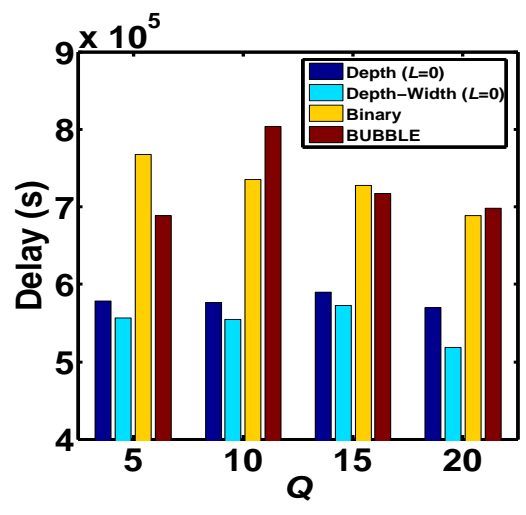

(c) $U=500$

Figure 8: Average latency comparison using the real world trace data when $L=0$ for our algorithms

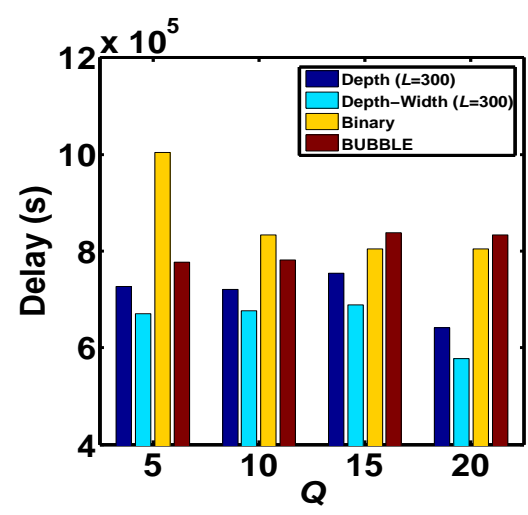

(a) $U=300$

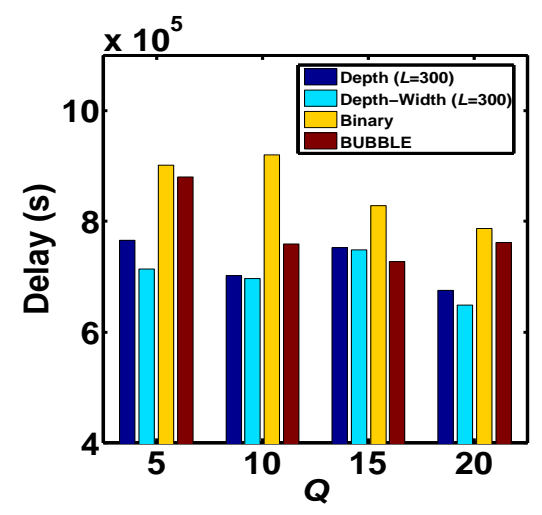

(b) $U=400$

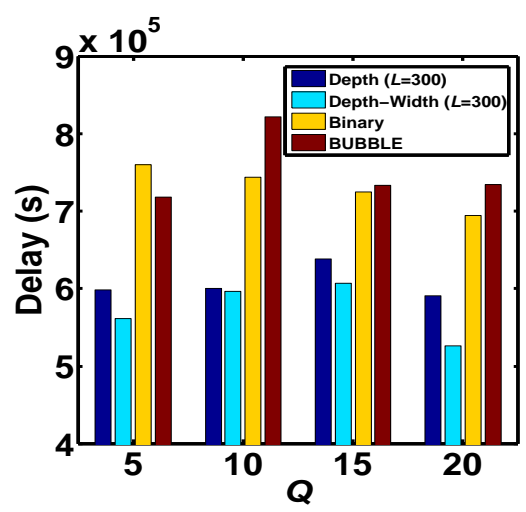

(c) $U=500$

Figure 9: Average delay comparison using the real world trace data when $L=300$ for our algorithms

[9] W. Gao and G. Cao, "User-centric data dissenmination in disruption tolerant networks," in INFOCOM, 2011, pp. 3119-3127.

[10] —, "On exploiting transient contact patterns for data forwarding in delay tolerant networks," in ICNP, 2010, pp. 193-202.

[11] P. Hui, J. Crowcroft, and E. Yoneki, "Bubbble rap: social-based forwarding in delay tolerant networks," IEEE Transactions on Mobile Computing, vol. 10, no. 11, pp. 1576 - 1589, 2011.

[12] E. M. Daly and M. Haahr, "Social network analysis for routing in disconnected delay-tolerant manets," in MobiHoc, Montreal, CA, 2007, pp. $32-40$.

[13] H. Dang and H. Wu, "Clustering and cluster-based routing protocol for delay-tolerant mobile networks," IEEE transactions on wireless communication, vol. 9, no. 6, pp. 1874-1881, June 2010.

[14] A. Vahdat and D. Becker, "Epidemic routing for partially connected ad hoc networks," Duke University, Technical Report CS-200006, April 2000.

[15] T. Spyropoulos, K. Psounis, and C. S. Raghavendra, "Spray and wait: an efficient routing scheme for intermittently connected mobile networks," in SIGCOMM workshop on delay-tolerant networking (WDTN), New York, USA, 2005, pp. 252-259.

[16] T. Spyropoulos, T. Turletti, and K. Obraczka, "Routing in delaytolerant networks comprising heterogeneous node populations," IEEE 
transactions on mobile computing, vol. 8, no. 8, pp. 1132-1147, August 2009.

[17] J. Burgess, B. Gallagher, D. Jensen, and B. N. Levine, "Maxprop: routing for vehicle-based disruption-tolerant networks," in INFOCOM, Piscataway, USA, 2006, pp. 1-11.

[18] S. Wang, M. Liu, X. Cheng, and M. Song, "Routing in pocket switched networks," IEEE Wireless Communications, vol. 19, no. 2, pp. 67-73, 2012.

[19] T. Henderson, D. Kotz, I. Abyzov, and J. Yeo, "CRAWDAD trace set dartmouth/campus/movement (v. 2005-03-08)," Downloaded from http://crawdad.cs.dartmouth.edu/dartmouth/campus/movement, March 2005.

[20] M. C. Gonzalez, C. A. Hidalgo, and A.-L. Barabasi, "Understanding individual human mobility patterns," Nature, vol. 453, no. 7196, pp. 779-782, June 2008.

[21] C. Song, Z. Qu, N. Blumm, and A. Barabási, "Limits of predictability in human mobility," Science, vol. 327, no. 5968, p. 1018, 2010.

[22] M. McNett and G. M. Voelker, "Access and mobility of wireless pda users," ACM SIGMOBILE mobile computing and communications review, vol. 9, no. 2, pp. 40-55, April 2005.

[23] T. Spyropoulos, K. Psounis, and C. S. Raghavendra, "Efficient routing in intermittently connected mobile networks: the single-copy case," IEEE transactions on networking, vol. 16, no. 1, pp. 63-76, February 2008.

[24] Y. Liao, K. Tan, Z. Zhang, and L. Gao, "Modeling redundancy-based routing in delay tolerant networks," in $C C N C$, Las Vegas, Nevada, USA, 2007, pp. $212-216$.

[25] K.P.Bogart, Introductory combinatorics. Academic Press, 2000.

[26] K. S. Trivedi, Probability and Statistics With Reliability, Queuing and Computer Science Applications. Wiley, 2002.

[27] T. Matsuda and T. Takine, "(p, q)-epidemic routing for sparsely populated mobile ad hoc networks," IEEE Journal on Selected Areas in Communications, vol. 26, no. 5, pp. 783-793, 2008.

[28] R. Groenevelt, P. Nain, and G. Koole, "The message delay in mobile ad hoc networks," Performance Evaluation, vol. 62, no. 1-4, pp. 210-228, 2005.

[29] G. Palla, I. Derényi, I. Farkas, and T. Vicsek, "Uncovering the overlapping community structure of complex networks in nature and society," Nature, vol. 435, no. 7043, pp. 814-818, 2005.

[30] E. P. Jones, L. Li, J. K. Schmidtke, and P. A. Ward, "Practical routing in delay tolerant networks," IEEE transactions on mobile computing, vol. 6, no. 8, pp. 943-959, August 2007.

[31] J. Leguay, T. Friedman, and V. Conan, "Evaluating mobility pattern space routing for dtns," in INFOCOM, Barcelona, Catalunya, SPAIN, 2006, pp. 1-10.

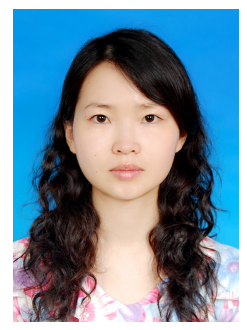

Shengling Wang received the $\mathrm{PhD}$ degree in computer science from Xi'an Jiaotong University, China, in 2008. After that, she worked at Tsinghua University as a postdoctor. Now, she is an associate professor at the Institute of Computing Technology, Chinese Academy of Sciences. Her current research interests include the mobility management, routing and load balancing in wireless and mobile networks.

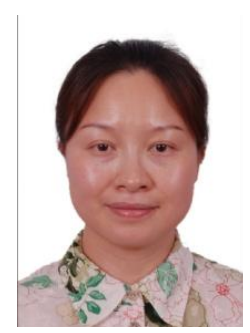

Min Liu received her B.S. and M.S. degrees in computer science from Xi'an Jiaotong University, China, in 1999 and 2002, respectively. She got her $\mathrm{Ph} . \mathrm{D}$ in computer science from the Graduate University of the Chinese Academy of Sciences in 2008. She is currently a professor at the Networking Technology Research Centre, Institute of Computing Technology, Chinese Academy of Sciences. Her current research interests include Mobility Management (including handoff management and location management), Wireless Resource Management, Delay $\backslash$ Disruptive-Tolerant Networks.

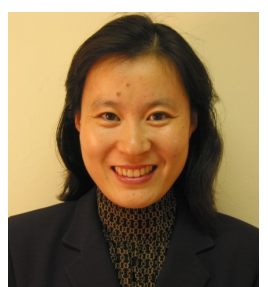

Xiuzhen Cheng received her MS and $\mathrm{PhD}$ degrees in computer science from the University of Minnesota - Twin Cities, in 2000 and 2002, respectively. She is an associate professor at the Department of Computer Science, The George Washington University, Washington DC. Her current research interests focus on cognitive radio networks, mobile handset networking systems (mobile health and safety), wireless and mobile computing, sensor networking, wireless and mobile security, and algorithm design and analysis. She has served on the editorial boards of several technical journals and the technical program committees of various professional conferences/workshops. She also has chaired several international conferences. She worked as a program director for the US National Science Foundation (NSF) from April to October in 2006 (full time), and from April 2008 to May 2010 (part time). She received the NSF CAREER Award in 2004. She is a senior member of IEEE and a member of ACM.

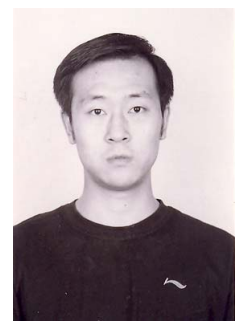

Jianhui Huang received the $\mathrm{PhD}$ degree in computer science from Xi'an Jiaotong University, China, in 2009. Now, he works at IBM as a software engineer. His current research interests include the mobility management, routing and cloud computing.

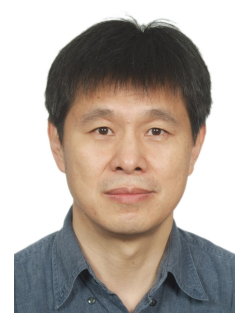

Biao Chen received his BS in Computer Science from Fudan University in China and MS in Mathematics and $\mathrm{PhD}$ in Computer Science from Texas A \& M University respectively. After graduation, he joined the Department of Computer Science in University of Texas at Dallas as a faculty member. Currently, he is a visiting professor in the Department of Computer and Information Science of University of Macau. His research interests include distributed systems, networking, and security. $\mathrm{He}$ is a member of Sigma Xi, IEEE, and ACM. 